\title{
Pasar por la carnicería: relatos de mujeres costarricenses sobre violencia obstétrica
}

\author{
Going through the carnage: Costa Rican women's stories about \\ obstetric violence
}

Melissa Hernández Vargas ${ }^{1}$

\begin{abstract}
RESUMEN
En este artículo se reflexiona sobre el impacto psicosocial de la violencia obstétrica y las manifestaciones más comunes experimentadas por siete mujeres costarricenses. Se realizó un estudio exploratorio-descriptivo de corte cualitativo, con un enfoque narrativo biográfico. Se utilizó la técnica de relatos de vida. Los principales resultados arrojan que la violencia obstétrica es una problemática palpable en los centros hospitalarios del país. Posee una alta complejidad y se presenta en todos los momentos de la atención (embarazo, parto y postparto). Además, existe un fuerte impacto psicosocial en la vida de las mujeres al experimentar estas violaciones a sus derechos y violencias dirigidas a sus cuerpos y psiques. La violencia obstétrica afecta de manera subjetiva, pero también a nivel relacional. Es importante generar cambios estructurales y culturales para reducir la naturalización de esta forma de violencia. Y, para erradicar la desigualdad y asimetrías de poder a nivel hospitalario y social.
\end{abstract}

Palabras clave: violencia obstétrica, impacto psicosocial, poder, desigualdad, privatización del daño.

\begin{abstract}
This article reflects on the psychosocial impact of obstetric violence and the most common manifestations experienced by seven Costa Rican women. An exploratory-descriptive qualitative study was carried out, with a biographical narrative approach. The life stories technique was used. The main results show that obstetric violence is a palpable problem in the country's hospitals. It is highly complex and occurs at all times of care (pregnancy, childbirth and postpartum). In addition, there is a strong psychosocial impact on the lives of women when experiencing these violations of their rights and violence directed at their bodies and psyches. Obstetric violence affects subjectively, but also at the relational level. It is important to generate structural and cultural changes to reduce the naturalization of this form of violence. And, to eradicate inequality and power asymmetries at the hospital and socially.
\end{abstract}

Key Words: obstetric violence, psychosocial impact, power, inequality, privatization of harm.

\footnotetext{
${ }^{1}$ Universidad de Costa Rica. Psicóloga social, estudiante de la Maestría en Estudios de las Mujeres, Géneros y
} Sexualidades. San José, Costa Rica. Correo electrónico: melissa.hernandezvargas@ucr.ac.cr

DOI: 10.15517/WL.V16I2.48101

Recepción: 20/2/2021 Aceptación: 25/5/2021 


\section{Introducción}

La violencia obstétrica es una manifestación dentro del amplio espectro de formas de violencia contra las mujeres. En términos académicos y políticos, el concepto surge debido al activismo feminista por la consecución de los derechos sexuales y reproductivos. Se materializa en el año 2006 en la ley venezolana Ley Orgánica sobre el Derecho de las Mujeres a una Vida Libre de Violencia (Arguedas 2014). Y, se extiende hacia otros países de la región latinoamericana como México y Argentina, quienes promulgan legislaciones.

En Costa Rica ha sido investigada por autoras y autores como Arguedas (2014 2016), Bolaños, Cerdas y Quesada (2017), Rodríguez y Vargas (2017), Campos (2018), Robles (2018), Salazar y Torres (2018), Hernández (2019) y Fuentes (2020). En el mes de febrero del 2020, la Asamblea Legislativa aprobó una reforma al artículo 12 de la Ley General de Salud en la que se contemplan ocho derechos que poseen las mujeres durante la atención obstétrica (Ruiz 2020, Ramírez 2020). Sin embargo, se considera que este esfuerzo requiere no sólo de una mayor divulgación, sino de una serie de cambios estructurales y culturales.

En el presente artículo interesa presentar los principales resultados producto de la elaboración de la tesis de licenciatura en Psicología de la Universidad de Costa Rica. Esta investigación se tituló "Impacto psicosocial de la violencia obstétrica a partir de relatos de vida de mujeres" (Hernández 2019). Pretendía construir una conceptualización del impacto psicosocial de la violencia obstétrica. Para esto, se entrevistó a siete mujeres costarricenses atendidas en hospitales públicos. Una por cada provincia del país. Las entrevistas se realizaron durante el año 2018 y la presentación de resultados se realizó el 26 de noviembre de 2019.

La investigación, en su totalidad, se posiciona desde los marcos teóricos proporcionados por la psicología social (Moscovici 1985, Martín-Baró 2000, Fernández 2009, Navarro 2012), la psicología política (Martín-Baró 2000, González 2004, González 2012), las teorías feministas (Lerner 1989, Crenshaw 1989, Amorós 2000, García 2003, Segato 2003, Federici 2004, Lagarde 2005, Varela 2008, De las Heras 2009, González 2010 
2013, De la Fuente 2013, Fernández 2013) y el enfoque de derechos humanos enfatizando en la defensa de la dignidad humana (Herrera 2008, Turégano 2009, Borja, García e Hidalgo 2011, Hendel 2017). Hay un interés académico y político en trabajar esta forma de violencia y comprender cómo influyen el poder y las relaciones estructurales en la vida de las mujeres. Además, hay una intención de descifrar cuáles son esas marcas psíquicas y psicosociales que quedan tras atravesar una experiencia deshumanizadora y que coloca a las mujeres en un lugar de exclusión.

A continuación, se presentará un breve recorrido por la metodología, el marco conceptual, los principales hallazgos y las conclusiones. Se hará énfasis en cómo entender y aplicar lo vivido por las mujeres en torno a los dos conceptos centrales de violencia obstétrica e impacto social.

\section{Metodología}

La pesquisa realizada posee un alcance exploratorio y descriptivo, logrado a partir del uso de una metodología cualitativa. Esta perspectiva permite extraer los significados que le asignan los sujetos a sus experiencias y presentar una descripción densa a partir de la experiencia de múltiples realidades (Salinas y Cárdenas 2009, Hernández et al 2014).

Medularmente tiene un enfoque biográfico narrativo al trabajar con experiencias reales de mujeres. El cual permitió conocer sus perspectivas, transiciones y cambios a partir de los procesos sociales e históricos ligados al contexto en el que se desarrolla la violencia obstétrica (Aceves 2001). Puntualmente se aplicó la técnica de relatos de vida a siete mujeres, quienes de manera electiva escogieron su seudónimo: Katrina, Susana, María, Mariana, Giselle, Dara y Sofía. El rango de edad iba de los 27 a los 36 años y poseían diferentes niveles de escolaridad, desde secundaria incompleta hasta universitaria completa. Cuatro de ellas tenían dos hijos o hijas y tres solamente uno o una.

Adicionalmente, se realizaron entrevistas a personas expertas y revisión de fuentes secundarias como herramientas para la obtención de información. Se considera que las vivencias particulares están intrínsecamente atravesadas por el contexto social e histórico en 
el que se habita, y es por esta razón que pueden dar pistas para entender fenómenos psicosociales.

Se realizó una transcripción y sistematización detallada de toda la información recolectada. Mientras que, el análisis de los datos se realizó a partir de dos técnicas: el análisis narrativo y el análisis comparativo. Estas permitieron contemplar cada relato por sí mismo y relacionarlos entre sí. A nivel ético se siguieron las recomendaciones del Comité Ético Científico de la Universidad de Costa Rica y las elaboradas por la OMS (1999) y Ellsberg y Heise (2007) para el trabajo de la violencia contra las mujeres. De manera insistente se buscó trabajar desde un marco de respeto y horizontalidad con las entrevistadas.

\section{Marco Conceptual}

\section{Delimitar la violencia obstétrica: una tarea difusa y un esfuerzo pendiente}

No existe un acuerdo tácito sobre cómo definir la violencia obstétrica, y esto se debe principalmente a su complejidad. Podría decirse que la forma en cómo esta se manifiesta puede llegar a responder a la cultura de cada país e incluso a las dinámicas de cada hospital o clínica que brinda atención obstétrica. Esto no implica que no existan modos de comprenderla o aspectos comunes en sus manifestaciones.

A partir de la lectura detallada de diferentes investigaciones se considera que han existido al menos cinco grandes categorías para su definición. La violencia obstétrica ha sido vista como una forma de violencia institucional, una violación a los derechos sexuales y reproductivos, negligencia médica, violencia de género y violencia contra las mujeres ${ }^{2}$. Aunque todas estas demarcaciones poseen relación directa con el fenómeno, desde una

\footnotetext{
2 Para ahondar en las descripciones de estas categorías, pueden revisarse los trabajos de Magnone (2011) y Cassiano et al (2016) (violencia institucional), Quevedo (2012), Belli (2013), García (2014), Castro (2014), Almonte (2016), Álvarez y Russo (2016) (violación a los derechos sexuales y reproductivos), Valdez et al (2013), Vergara (2014), Castro (2014) (negligencia médica), Villegas (2009), Quevedo (2012), Aguirre et al (2013), Belli (2013), Camacaro (2013), Machado (2014), Bellón (2015) (violencia de género), Magnone (2011), Fernández (2013), Villanueva et al (2016), Pereira (2017), Rodríguez y Aguilera (2017), Lira (2018) (violencia contra las mujeres).
} 
mirada feminista es importante subrayar que la violencia obstétrica es una forma de violencia ejercida contra las mujeres.

¿Por qué? Porque implica el reconocimiento de la naturalización de la desigualdad que se ha dado a partir del control y la represión de los cuerpos de las mujeres y sus deseos. Afirma que el patriarcado como estructura social legitima actos de discriminación contra estas y atenta contra su acceso a los derechos sexuales y reproductivos, así como a la salud reproductiva. Y, porque, al igual que con otras formas de violencia, suele existir una victimización y culpabilización de las mismas generando repercusiones directas en sus psiquis.

Es menester insistir en que la violencia obstétrica es una forma de violencia contra las mujeres porque ha estado relegada al silenciamiento cultural durante siglos. Ha sido invisibilizada y naturalizada en la disciplina y atención obstétrica, llegando incluso a ser parte de las dinámicas y jerarquías que se dan a lo interno de las instituciones. Es importante erradicarla.

Más allá de esto, su reconocimiento social ha sido escaso y permite que, en palabras de Hernández, se busque "homogenizar la vivencia de la feminidad, se crea una visión del cuerpo femenino como simple máquina que realiza de manera mecánica el acto de parir y se anula su capacidad de decisión junto a la posibilidad de generar conocimiento sobre los procesos que atraviesa en estos momentos" $(2019,121)$.

Como punto de partida, en esta investigación se utilizó la definición de Gabriela Arguedas que señala que la violencia obstétrica es:

un conjunto de prácticas que degrada intimida y oprime a las mujeres y a las niñas en el ámbito de la atención en salud reproductiva y, de manera mucho más intensa, en el período del embarazo, parto y postparto $(2014,146)$.

Adicionalmente, se consideró importante la puntualización de Laura Belli, quien señala que la violencia obstétrica es ejercida por "el personal de salud sobre el cuerpo y los procesos reproductivos de las mujeres expresada en un trato deshumanizado, un abuso de medicalización y patologización de los procesos naturales" (2013, 29-30). Es importante 
reconocer que el personal administrativo también puede ejercer violencia obstétrica e incluso se da con alta frecuencia.

Tanto el concepto de Arguedas (2014) como el de Belli (2013) destacan el lugar de la opresión y el contexto en el que se da la violencia. Permiten visibilizar el espacio físico y simbólico en el cual se desarrolla la violencia obstétrica y comprender que de ninguna manera es algo natural, sino socialmente construida a partir de jerarquizaciones, abusos de poder e ideas culturales sobre la maternidad y la atención de los procesos reproductivos.

\section{Impacto psicosocial o las huellas de la violencia obstétrica}

El principal aporte de esta investigación fue la construcción del concepto de impacto psicosocial de la violencia obstétrica. Tomó como base los conceptos de trauma psicosocial (Martín-Baró 1990) y daño psicosocial (Simoes 2014, Dobles y Rodríguez 2019). A diferencia de estas definiciones, el impacto psicosocial no sólo recupera los efectos generadores de sufrimiento, sino también los recursos y formas de resistencia que poseen las mujeres. Según Hernández puede definirse como:

Las consecuencias psicosociales que son producto de una experiencia que ha causado un quiebre o interrupción en la vida de una persona y su contexto inmediato, incluyendo sus relaciones de pareja, familiares o comunitarias, así como otras establecidas en espacios de carácter laboral, educativo u otro. Estas consecuencias psicosociales pueden ser cambios, lesiones o huellas visibles o invisibles a nivel físico, emocional, psicológico, subjetivo, salud o relacional, pero también pueden implicar el fortalecimiento de lazos afectivos, accesibilidad a recursos, formas de resistencia y aumento de redes de apoyo. Su intensidad varía de una persona a otra según los recursos personales y colectivos con los que cuente $(2019,192-193)$.

Debe notarse que este concepto reconoce tanto aspectos de carácter individual como colectivos. En palabras sencillas buscó identificar qué pasaba en la vida de las mujeres a nivel 
subjetivo y a nivel relacional al experimentar eventos de violencia obstétrica. En el próximo apartado se puntualizará cuáles fueron los hallazgos en torno a este concepto.

\section{Hallazgos}

Si bien es cierto el sistema de salud costarricense posee múltiples fortalezas y una amplia cobertura a lo largo del país, no está exento de denuncias sobre las situaciones de violencia que se han dado en la atención de la salud reproductiva (Oviedo 2015, Chaves 2015, Morris 2015). Las quejas han tomado protagonismo en los últimos años, pero sin duda las prácticas de discriminación y violencia que se dan en estos espacios poseen raíces de larga data.

Seguidamente se presentarán los principales hallazgos en torno a la violencia obstétrica, las relaciones de poder inmersas en la misma y el impacto psicosocial identificado en los relatos de vida de las mujeres entrevistadas.

\section{Relatos desde la carnicería: la violencia obstétrica}

La violencia obstétrica es vivenciada por las mujeres como una experiencia traumática, abusiva, deshumanizada y que las coloca en un lugar de animalidad, inclusive. En palabras de una de las entrevistadas "no hay forma de decirlo, para ellos somos un chanchito que están destazando y ya" (Giselle, comunicación personal, 24 de agosto de 2018).

Uno de los propósitos de la investigación era identificar las manifestaciones de violencia obstétrica que habían enfrentado las mujeres entrevistadas. Como punto de partida debe señalarse que todas experimentaron malos tratos durante al menos dos momentos de la atención, a saber, embarazo, parto y postparto. Inclusive algunas de ellas en los tres períodos.

A diferencia de otras investigaciones las mujeres reportaron más manifestaciones durante el postparto, lo cual es un dato importante porque en este período ellas suelen ser 
"olvidadas" e ignoradas por el personal una vez que nace su hijo o hija. Es necesario reconocer las situaciones que acontecen en este período como una forma de violencia porque visibiliza los aspectos simbólicos que subyacen a la violencia obstétrica. Por ejemplo, que esta es una expresión del patriarcado y que en cada una de sus manifestaciones sale a relucir la asimetría y desigualdad de poder entre ellas y el personal a lo largo de la atención (Hernández, 2019). Esta desigualdad se refleja en el siguiente relato:

Vos entrás ahí en un limbo raro. Donde la gente va y viene (...) Te atiende uno. Te atiende otro. No sabés cómo se llama nadie. No sabés qué puesto tiene nadie. Entonces uno no sabe a quién acudir (...) Y uno dice: "Esta muchacha no está haciendo las cosas como conviene. ¿Pero entonces a quién le digo que lo haga mejor?” (Susana, comunicación personal, 29 de agosto de 2018).

Sobresale la incomodidad de las mujeres frente a estas dinámicas disímiles, pero a la vez, su impotencia frente a la estructura misma. Esta sensación se repite en los demás relatos.

Ahora bien, a modo de caracterizar las experiencias de las mujeres y las manifestaciones de violencia obstétrica atravesadas se utilizó la división de la OMS (2014) que identifica cinco subtipos de violencia obstétrica. Son:

\section{Intervenciones y medicalización innecesarias y de rutina}

Las mujeres señalaron haber sido expuestas al uso de medicación y tecnologías que aceleraban el proceso de parto. Como señala Almonte (2016) esta aplicación tiende a homogeneizar los cuerpos de las mujeres y patologiza los procesos reproductivos. El principal problema es que se han utilizado no sólo en los casos en que se requiere, sino que se usan de manera rutinaria generando una sobreintervención que solamente favorece al personal obstétrico y el desarrollo de sus labores. Pareciera que el hospital se transforma en una fábrica y su único objetivo es acelerar los procesos de producción:

Me dice: "¿Usted no siente nada?". "No. Yo no estoy sintiendo nada". "Con la cantidad de suero que le estoy poniendo, usted debería de estar 
Wimblu, Rev. Estud. de Psicología UCR, 16(2) 2021 (Julio-Diciembre): 93-118 /ISSN: 1659-2107

revolcándose en esa cama" (Susana, comunicación personal, 29 de agosto de 2018).

Para las mujeres incomoda el hecho de que no se les brinde información sobre los efectos de estas intervenciones e incluso en el caso de Dara se llegó a poner su vida en riesgo. Sin embargo, reconocen que en el momento sienten una incapacidad de oponerse a estas intervenciones. Esta imposibilidad de reclamo tiene una raíz cultural desde la mirada de Paula Quevedo (2012), quien resalta que hay un fuerte reconocimiento y un lugar social de la medicina como acreedora del conocimiento para intervenir sobre los cuerpos. De manera imaginaria se considera que una persona debe estar sumamente agradecida por la atención que le brinda el o la profesional en salud como si fuera un favor.

\section{Maltrato, humillaciones, agresión verbal o física}

En esta categoría se recopilaron una serie de manifestaciones que destacan por su diversidad e impacto directo sobre los cuerpos de las mujeres. Van desde la violencia física, violencia verbal hasta la violencia psicológica. Cabe resaltar que son formas de violencia más fáciles de reconocer, ya que a nivel social suele hablarse de ellas en medios de comunicación, centros educativos u otros espacios.

Pareciera que a través de ellas existe una necesidad de regular los cuerpos de las mujeres tanto desde la emisión de juicios sobre su forma de ser, pero también afectándolas directamente al generar daño físico o psíquico:

Me ponían a hacer sentadillas (...) Con esa panza, pesaba casi 100 kilos. Con esa panza, que yo ya ni me la aguantaba (Mariana, comunicación personal, 18 de julio de 2018).

(...) me rompió el borde de la vagina (...) Yo no sé si fue a propósito (...) Donde hace el tacto, el mae como que revienta y rompe (María, comunicación personal, 29 de agosto de 2018). 
Ambas citas exponen situaciones directas sobre los cuerpos de Mariana y María, otros relatos exponen actos de violencia física como punzadas o cicatrices por inyecciones, moretes en los brazos, control y regulación de los movimientos del cuerpo. Estas secuelas generan marcas en la piel o en los órganos de las mujeres. Verlas o sentirlas hace que el recuerdo de la violencia obstétrica aparezca una y otra vez. Son fuentes de malestar que pueden extenderse hasta meses después de la vivencia.

En el plano verbal las mujeres reportaron actos de discriminación, expresión de estereotipos hacia personas extranjeras, reclamos hacia ellas por sus reacciones, expresar sentimientos o hacer preguntas, comentarios sobre su sexualidad y que se les daba órdenes de manera violenta e imperativa:

"Usted no tiene los senos adecuados, por lo tanto, usted no es una madre". "No vas a satisfacer con las condiciones necesarias que ocupa su hijo para poder alimentarse" (María, comunicación personal, 29 de agosto de 2018).

El dolor tiene que aguantarlo uno callado, porque no puede gritar, porque a él le molesta que una mamá grite y se exprese (Giselle, comunicación personal, 24 de agosto de 2018).

De repente, se espera que las mujeres sean sujetas pasivas desde el momento en que ingresan a los centros hospitalarios. Como señala Arguedas (2016) tampoco se les reconoce como sujetos cognoscentes, ni sujetos morales. Las frases emitidas por el personal se transforman en violencia psicológica que incide en su subjetividad. Todas las

manifestaciones ya citadas, así como los juicios hacia sus modales o comportamientos e incluso la violencia psicológica durante la lactancia, hablan de un lugar de infantilización en el que son colocadas. Y, exponen la discriminación y culpabilización a las que son sometidas.

\section{Falta de insumos o instalaciones adecuadas}

Las entrevistadas manifestaron atravesar situaciones en las que se limita su acceso a una atención de calidad y a derechos básicos como el acceso a la salud. Van desde carencias en insumos, falta de recursos, malas condiciones de infraestructura, exposición a condiciones 
insalubres y la negación de prestación de servicios de atención, alimentación o rechazo de procedimientos como el salpin. Algunos ejemplos desde sus relatos son:

Hice las vueltas, no me quisieron hacer las vueltas porque estoy muy joven. Vos no tenés derecho a decir en qué momento no más hijos. Ni te los van a mantener tampoco, verdad (...) Para que te puedan hacer el salpin tu esposo tiene que estar de acuerdo. Y tampoco. O sea, el cuerpo es mío, pero también mi esposo (Mariana, comunicación personal, 18 de julio de 2018).

Éramos once mamás y solo había un sillón, entonces lo que ellas hacían...y, para variar, de las once, siete éramos de cesárea. Las otras eran partos normales. Entonces las de parto normal se sentaban o dormían en el piso y el sillón en teoría era nada más para la gente que éramos de cesárea, pero di, un sillón para siete, imposible (Sofía, comunicación personal, 7 de setiembre de 2018).

Al leer ambas narraciones es inevitable no pensar en actos de tortura a los deseos y necesidades de las mujeres. Estas manifestaciones reflejan las carencias que existen a nivel institucional como la falta de recursos, malas condiciones de infraestructura, pero también, resaltan el hecho de que las mujeres no reciben una atención adecuada, ni mucho menos digna. Se está limitando su acceso a una gran cantidad de derechos como la salud integral y sus derechos sexuales y reproductivos.

\section{Ejercicios de residentes y practicantes sin autorización de las mujeres}

Actos como la realización de múltiples tactos vaginales por diferentes personas que integran el personal médico, de enfermería y estudiantes son intervenciones frecuentes que se realizan sin el consentimiento de la mujer lo cual genera una sensación de abuso y dolor.

A las mujeres se les objetiviza al punto de reducirlas a ser un "cuerpo" o "cosa" que puede ser explorado por personas desconocidas: 
Wimblu, Rev. Estud. de Psicología UCR, 16(2) 2021 (Julio-Diciembre): 93-118 /ISSN: 1659-2107

Pasó un grupo de doctores como a las seis de la tarde. El ginecólogo de turno con 10 estudiantes. Nada más: "Abra las piernas”. Y todos hicieron el tacto. ¡Todos! (grita). O sea, imagínate. Y yo me acuerdo que a mí me dolía (...) "Mamá, ¡ellos son estudiantes necesitan aprender!”. Ni siquiera. Ya yo después vi que ellos tenían que pedir mi autorización, y yo tenía que decidir si sí o no (Mariana, comunicación personal, 18 de julio de 2018).

\section{Discriminación por razones culturales, económicas, religiosas y étnicas}

La violencia obstétrica se entremezcla con otras formas de violencia simbólica que reproducen relaciones de dominación, desigualdad, exclusión y discriminación (Restrepo et al 2016). Estas son problemáticas que agravan la vulnerabilidad y el acceso a derechos (Arguedas 2016). En el caso de las mujeres si ya son dejadas de lado por su condición genérica, sumando una o varias de estas características se profundiza su exposición a la violencia obstétrica.

En los relatos de las mujeres consultadas se señaló haber vivido discriminación por su condición migratoria, edad y por razones económicas:

La gente tiene como un repudio, como una cosa, por las adolescentes y por las muchachas jóvenes embarazadas que dan a luz (...) O sea, uno dice, se abusan de que son chiquillas. Porque no tienen quién las defienda. (Mariana, comunicación personal, 18 de julio de 2018).

La violencia simbólica les deja en completa desventaja frente al personal que les atendió. Aún más allá, provoca un mayor aumento de los malos tratos y más impunidad frente a estos.

\section{Relaciones de poder como constituyentes de la violencia obstétrica}

Existe una correlación dialéctica entre el poder y la violencia obstétrica, ya que este suele ser medular en las relaciones que se establecen dentro de las dinámicas hospitalarias y 
en el espacio médico obstetra. Para Foucault (1998) el poder es parte de las relaciones sociales, pero no es exclusivo de un solo grupo o persona. Todas y todos ejercen poder en alguna medida, lo cual implica que las relaciones de poder son intencionales y poseen un objetivo. Se expresan en la cotidianidad y atraviesan tanto los cuerpos como los pensamientos.

Posee dos caras que pueden delimitarse a nivel teórico-conceptual. La primera de ellas es que el poder es usado para controlar y dominar. Como se ha venido insistiendo, en una sociedad patriarcal el poder se materializa en diferentes formas de dominación y control hacia las mujeres, sus cuerpos, sus comportamientos y sus psiques. De la Fuente (2013) señala que el poder en su forma de dominación genera una gran cantidad de sufrimiento y presenta múltiples dificultades para su erradicación debido a su legitimación. Una de estas manifestaciones es el control violento y jurídico de la sexualidad, la capacidad reproductiva y el cuerpo femenino.

En el caso hospitalario, estas especificaciones se expresan tanto en el nacimiento de la obstetricia como disciplina a cargo de los procesos reproductivos, ya que copta el conocimiento para realizar las intervenciones asociadas a estos procesos, como en las jerarquías en las interacciones entre el personal y las mujeres (Camacaro 2008, Machado 2014). Sobre el primer tópico es menester señalar que históricamente se genera una disputa entre la disciplina médico-obstétrica y la labor que realizaban las parteras. Culmina con el rechazo a las prácticas que ejecutaban estas mujeres (Federici 2004, Belli 2013, Arguedas 2014). Acerca del segundo punto, las entrevistadas expresan:

Pero supongo que tiene que haber sido algún doctor, o algún supervisor o el jefe de alguien. Porque llamaron a la muchacha que me había asistido en el parto (...) Y la muchacha tenía gesto de una persona a la que se estaba regañando. Porque estaba cabizbaja, con las manos en los muslos. Ella bajaba la cabeza. Y la persona que estaba con ella hablándole de frente le golpeaba la mesa. Levantaba las manos. De viaje se notaba que era una conversación fuerte o que le estaban llamando la atención o regañando (Susana, comunicación personal, 29 de agosto de 2018). 
También, las mujeres narran eventos en los cuales se refleja una pugna dentro del mismo personal por demarcar quién es la autoridad, quién sabe más y cuáles deben ser los roles a respetarse. Giselle da un ejemplo claro, cuenta que ante las dificultades que surgieron antes del parto, una enfermera obstetra le dice que puede ayudarle a reventar la fuente para acelerar el proceso de parto, pero un médico obstetra que observaba la interacción le dice que: “(...) ella no tenía que meterse en eso. Porque ella no tenía el título para hacer eso" (Giselle, comunicación personal, 24 de agosto de 2018). Se crea una disputa entre ambos dejando de lado las necesidades de Giselle, quien ya llevaba cuatro días en labor de parto y solamente cinco centímetros de dilatación. El médico insiste en que ella puede parir por vía vaginal, y tiempo después admite ante otro médico de rango superior que sí es necesaria una cesárea.

Esta misma demarcación de la autoridad y la jerarquización la realiza el personal con las mujeres. Giselle resalta dos símbolos que, desde su perspectiva, el personal utiliza para remarcar la diferencia. El primero de ellos es el título universitario y el segundo, trabajar dentro de una institución pública. Por su parte Sofía comenta:

Uno dice: “¿Quién tendrá la razón?”. Uno me dice que va a ser cesárea y otro me dice: "Se los provoco y va a ser más rápido". Entonces yo llegué y le dije a él: "No, yo prefiero la cesárea". Él me dijo: "Usted no sabe a lo que va". Yo le dije: "No. Yo ni sé qué es un parto normal, ni que es una cesárea, porque nunca he tenido hijos". Y me hace: "Sí, pero es que la cesárea duele más” (...) pero él nunca me explicó ni qué. O sea, ni me tocó (Sofía, comunicación personal, 7 de setiembre de 2018).

Como puede verse uno de los espacios más disputados por el personal es aquel que corresponde al plano del saber. De manera insistente recalcan que son acreedores del conocimiento más óptimo para atender los procesos reproductivos, aun cuando las mujeres expresen sensaciones en sus cuerpos o reaccionen de manera instintiva frente a un evento. Arguedas (2016) califica estas dinámicas como una aplicación del poder disciplinario y una injusticia epistémica, en la que las mujeres son despojadas de su capacidad de pensar. 
Por otro lado, la segunda cara del poder es la resistencia, por esto puede decirse que este es siempre relacional y que viene "desde abajo" (Foucault 1998). A pesar de la desigualdad que viven, las mujeres nunca son meras receptoras de la violencia. Compartieron sus pequeños y grandes gestos parar hacer cara a lo que acontecía en los hospitales. Reaccionaron con enojo, buscaron ser más amables con el personal, aunque esto no reducía los malos tratos y usaron el silencio como arma. Al salir de la institución realizan denuncias, se niegan a volver a ser madres, hablan sobre sus experiencias con personas cercanas y se cuestionan los mandatos sobre la maternidad que les fueron transmitidos (Hernández, 2019).

\section{Salir de la carnicería: el impacto psicosocial}

Puede afirmarse que existen diversos momentos en los que se puede reconocer la estela del tener que atravesar una experiencia de violencia obstétrica. Anteriormente se expuso una pincelada del durante y en seguida se mencionará qué sucede con el después. Justo este es el aspecto medular del concepto de impacto psicosocial, que como se mencionó anteriormente, busca recuperar las consecuencias psicosociales de atravesar una situación de violencia que viene a causar una interrupción en la vida de las mujeres.

Frente a la imposibilidad de analizar cada una de las consecuencias psicosociales detectadas a lo largo de estas páginas, mencionaré aspectos medulares trabajados en la investigación. Un primer punto es que para las mujeres es más sencillo identificar los impactos en aquellos niveles de carácter más individual (subjetivo, psicológico, emocional, físico y sexual). Se considera que esto tiene raíz en una privatización del daño en la que a nivel social suele asignarse una responsabilidad individual para cargar con el sufrimiento (Centro Nacional de Memoria Histórica 2014). Esto se agrava en los casos de violencia llegando al punto de culpabilizar o victimizar a quienes atraviesan una de estas situaciones.

De manera concreta, algunas de las consecuencias psicosociales que se lograron identificar a partir de los relatos son: 
Wimblu, Rev. Estud. de Psicología UCR, 16(2) 2021 (Julio-Diciembre): 93-118 /ISSN: 1659-2107

Tabla 1. Impacto psicosocial de la violencia obstétrica según las mujeres entrevistadas

\begin{tabular}{|c|c|}
\hline Nivel & al \\
\hline Subjetivo & $\begin{array}{l}\text { - Fragilidad y desamparo propios. } \\
\text { - Tristeza por no vivir una experiencia de calidad. } \\
\text { - Disminución o desaparición del deseo de ser madres de nuevo. }\end{array}$ \\
\hline Emocional & $\begin{array}{l}\text { - Emociones intensas y extremas (tristeza, enojo, miedo, cansancio, soledad). } \\
\text { - Reaparecen al recordar y poseen repercusiones en sus relaciones. }\end{array}$ \\
\hline Psicológico & $\begin{array}{l}\text { - Cambios en el comportamiento. } \\
\text { - Manifestaciones psicosomáticas. } \\
\text { - Aislamiento. } \\
\text { - Aumento de consumo de alimentos. }\end{array}$ \\
\hline Físico & $\begin{array}{l}\text { - Marcas y cicatrices. } \\
\text { - Cambios en la imagen corporal que afectan la autoestima. } \\
\text { - Complicaciones de salud como no contener la orina, dolor, hemorragias u otras. }\end{array}$ \\
\hline Sexual & $\begin{array}{l}\text { - Dificultad para retomar relaciones sexuales. } \\
\text { - Aumento de consumo de métodos anticonceptivos. }\end{array}$ \\
\hline $\begin{array}{ll}\text { Laboral } y \\
\text { educativo }\end{array}$ & $\begin{array}{l}\text { Los cambios reportados no fueron significativos, ya que hubo mucha diversidad en las } \\
\text { condiciones de las mujeres. Un par de ellas mencionó que sí cambió su rendimiento e incluso } \\
\text { una de ellas renunció a su trabajo. }\end{array}$ \\
\hline $\begin{array}{ll}\text { Pareja } \quad y \\
\text { familia }\end{array}$ & $\begin{array}{l}\text { En algunos casos puede haber conflictos y peleas, pero también un aumento del apoyo en el } \\
\text { cuido y las labores domésticas. }\end{array}$ \\
\hline Social & $\begin{array}{l}\text { - Deshumanización de las mujeres. } \\
\text { - Normalización e institucionalización de la violencia. } \\
\text { - Desestabilización de la vida cotidiana. } \\
\text {-Cambios en la subjetividad e identidad. } \\
\text {-Desconfianza en el sistema de salud pública. } \\
\text { - Aprendizaje vicario, desesperanza e impotencia. } \\
\text { - Violación de derechos sexuales y reproductivos. } \\
\text { - Aumento del desconocimiento de las mujeres sobre su cuerpo, su sexualidad y la } \\
\text { reproducción. } \\
\text { - Fortalecimiento de vínculos. } \\
\text { - Identificación con otras mujeres y apoyo. }\end{array}$ \\
\hline
\end{tabular}

Fuente: Elaboración propia a partir de Hernández (2019), Impacto Psicosocial de la violencia obstétrica a través de relatos de vida de mujeres. 
Sale a relucir la enorme diversidad de impactos que genera la violencia obstétrica, y es posible discutir que ante la invisibilización de los mismos es necesario criticar la idea de que los malestares de las mujeres son parte de la misma condición de "ser mujer" o que son un sacrificio. Incluso todo el proceso de embarazo, parto y postparto se asocia al dolor. Es imperante reconocer que esta forma violencia posee raíces sociales y su impacto también produce consecuencias a este nivel como las citadas en la Tabla 1. Una apuesta posible para enfrentar esta privatización sería ingresar en una lógica colectiva para hacer frente a estas huellas de la violencia. No debe dejarse que sea vivida y procesada en soledad y aislamiento.

Por otro lado, es de suma importancia no entender el impacto psicosocial como una marca de la cual las personas no pueden desprenderse, ni tampoco tomarlo como una medición del sufrimiento, ni mucho menos asumir que es vivido de la misma manera por todas las personas. A su vez, el concepto reconoce aquellas consecuencias que son vividas en la inmediatez, pero también las que se extienden durante meses o incluso años (Hernández 2019).

Insistir en que la violencia obstétrica deja huellas a nivel psicosocial implica entender que las mujeres son una colectividad que ha sido históricamente oprimida y que esta forma de violencia ha sido sufrida por estas desde hace décadas. Continúa sucediendo e incluso es vista como parte del proceso de atención. Ahora, esta colectividad de mujeres posee múltiples diferencias en su interior como su condición de clase, etnia, condición migratoria, edad u otras (Arguedas 2016, Hernández 2019). Estos contrastes inciden en las formas de violencia que experimenta cada mujer y en sus posibilidades para hacer frente a la circunstancia.

Más allá de que el ejercicio de esta violencia pueda ser personalizado por las mujeres y se señale alguna persona particular que lo ejerza, es necesario visibilizar que existe una institución y un estado que falla en la garantización del principio de la dignidad humana. Las mujeres se enfrentan de golpe con el sistema patriarcal y su encasillamiento en determinadas subjetividades femeninas. La violencia obstétrica se vuelve una forma de asegurar y regular los comportamientos sumisos, pero ninguna mujer puede o debe cumplir con este mandato (Arguedas 2014, Hernández 2019). 


\section{Conclusiones}

Pasar por la carnicería: “como es primeriza, déjenla batallar, “¿para qué se ponen a tener niños?", "su cuerpo fisiológicamente no está apto. No tiene condiciones para tener un hijo natural", "y me punzaba, y me punzaba, y me punzaba. Y yo ya no aguantaba" (Mariana, Dara, María y Katrina, comunicación personal, 2018).

Pasar por la carnicería: "le voy a provocar los dolores. Eso es rápido. Le voy a poner un suerito y ya", "la lactancia en el hospital es una cosa pavorosa. La muchacha me agarraba de atrás. Me empujaba el brazo. Empujaba a Helena. Me presionaba contra la bebé” (Sofía y Susana, comunicación personal, 2018). En resumidas cuentas "para él uno no tiene derecho de gritar. No tiene derecho de expresarse. Nada más va a la carnicería. Le abren la cesárea o el piquete. Y tiene que aguantar. Porque él es el profesional y uno no sabe" (Giselle, comunicación personal, 24 de agosto de 2018).

Esta investigación confirma que la violencia obstétrica es una realidad en los hospitales públicos costarricenses y es necesario reconocerla como tal. No sólo expone la violación a derechos humanos de las mujeres, sino que deja huellas en sus cuerpos y psiques.

Es una forma de violencia multidimensional, que varía según el contexto histórico e incluso las dinámicas que se dan en cada hospital. En esta pesquisa se encontró que en provincias como Limón o Puntarenas las experiencias de las mujeres fueron más crudas y reflejan mayores carencias en cuanto a la infraestructura y las condiciones propias de los hospitales (Hernández 2019). Además, hay una enorme cantidad de situaciones que pueden ser catalogadas dentro de este concepto y debe investigarse más aquellas que se presentan en el período de postparto.

De igual manera, esta forma de violencia contra las mujeres es una expresión del patriarcado. Reproduce y refuerza estereotipos sobre la "mujer", sostiene la desigualdad genérica y despoja a las mujeres de los conocimientos propios sobre su cuerpo, la sexualidad y la reproducción (Camacaro 2008, Quevedo 2012, Arguedas 2014, Restrepo et al 2016). Muchas veces sus manifestaciones son utilizadas como un castigo cuando las mujeres no cumplen con los roles esperados y expresan su capacidad de decisión. 
Existe un contexto posibilitador para la ocurrencia de estos eventos que se desarrolla no sólo en las instancias físicas de los hospitales, sino también se caracteriza por una legitimación a nivel cultural y macrosocial. Tanto la disciplina obstétrica como el Estado fallan a la hora de garantizar el bienestar de las mujeres.

Como ha sido resaltado por otras investigaciones y fue constatado en la presente, el tema del poder es central en el acontecer de la violencia obstétrica (Belli 2013, Arguedas 2014 2016). Muestras de esto son su presencia en las relaciones e interacciones dentro del hospital, así como en la aplicación de procedimientos y el uso del conocimiento. Lamentablemente esta repartición del poder viene desde la experiencia educativa del personal, se materializa en la jerarquización que posee el mismo cuerpo obstétrico y reproduce un orden social en el que las mujeres están en el último lugar.

Frente a esto las mujeres optan por realizar pequeñas acciones de resistencia como las narraciones compartidas. Acto que se vuelve de doble filo porque funciona como una advertencia hacia otras mujeres dentro de la familia, pero perpetua un silenciamiento del tema a nivel social. Sin querer, en muchas ocasiones se accede a reafirmar el pacto patriarcal (Hernández 2019). Otras operaciones que realizan dentro del hospital son ser amables esperando que se reduzca la violencia, seguir las recomendaciones de otras mujeres y usar el silencio como acto de resistencia. También, realizan denuncias legales o verbales y se cuestionan los mandatos sociales asociados a ser "mujer" o "madre".

En otros términos, se evidenció que las mujeres experimentan una gran cantidad de manifestaciones de violencia obstétrica que van desde formas sutiles hasta otras más directas. Puede realizarse un análisis de cada una de estas formas, pero en general, muestran la necesidad de acelerar los procesos de parto, asegurar un control e instrumentalización de los cuerpos de las mujeres, dejan marcas a nivel físico y psíquico, infantilizan, culpabilizan, limitan el acceso a la salud, reproducen una moralidad estereotipada sobre cómo deberían ser las mujeres, entre muchos otros intereses (Hernández 2019).

Estas situaciones producen un impacto psicosocial que deja huellas en las mujeres y en las personas que conforman sus redes, extendiéndose incluso al plano social. Como se apuntó hay que eliminar la concepción de la privatización del daño e incluso ahondar más en 
la investigación de cómo afecta la violencia obstétrica a las parejas o familiares con cercanía a las mujeres. Se requiere una mirada psicosocial para la comprensión de este fenómeno y dejar de repetir visiones psicologistas tanto para su entendimiento como su atención y prevención.

¿Qué se puede hacer? Validar y visibilizar esta forma de violencia, no contribuir a la privatización del daño, promover cambios socioculturales e institucionales para reducir la naturalización e invisibilización de estos actos, realizar campañas informativas, informar a las mujeres sobre sus derechos e impulsar su cumplimiento, y brindar acompañamiento a las mujeres que atraviesan una situación de este tipo (Hernández 2019). Es importante visibilizar todos estos acontecimientos para propiciar que tanto las mujeres como sus familias o acompañantes cuenten con información para identificar la violencia, conozcan sus derechos y posibilidades de denuncia. Debe discutirse de qué manera se pueden generar cambios reales en las disciplinas asociadas a los procesos de reproducción y en la vida cotidiana dentro de los hospitales. Y, sin duda alguna, debe apostarse por cambios socioculturales que apunten hacia la erradicación de la violencia obstétrica y de todas las formas de violencia contra las mujeres.

Ninguna mujer es una máquina, ni un objeto. No debe ser reducida a una condición de animalidad, ni debe verse sometida a malos tratos o torturas. Estas páginas son una invitación para que, desde la academia, el activismo y el profesionalismo se pueda trabajar desde una ética feminista y partir de una perspectiva no psicologista o patologizante. Desde una corresponsabilidad social cabe repetir la pregunta de Arabella Salaverry “¿quién repara el daño de esta práctica obscena?” $(2016,59)$.

\section{Referencias}

Aceves, Jorge. 2001. «Experiencia biográfica y acción colectiva en identidades emergentes». Revista Espiral, 7, (20): 11-37. http://148.202.18.157/sitios/ publicacionesite/pperiod/espiral/espiralpdf/Espiral\%2020/3-29.pdf

Almonte, Gabriela. «Violencia ginecológica y obstétrica. La medicalización del cuerpo femenino». Revista Décsir EAM, 2 (2016): 1-10. http://decsir.com.mx/wpcontent/uploads/2016/03/1-indagaciones.pdf 
Amorós, Celia. 2000. Feminismo y filosofía. Madrid, España: Editorial Síntesis.

Arguedas, Gabriela. 2014. «La violencia obstétrica: propuesta conceptual a partir de la experiencia costarricense». Cuadernos Intercambio sobre Centroamérica y el Caribe, 11, (1): 145-169. DOI 10.15517/C.A..V11I1.14238.

— 2016. «Reflexiones sobre el saber/ poder obstétrico, la epistemología feminista y el feminismo descolonial, a partir de una investigación sobre la violencia obstétrica en Costa Rica». Revista Solar, 12, (12): 65-89. DOI. 10.20939/solar.2016.12.0105

Belli, Laura. 2013. «La violencia obstétrica: otra forma de violación a los derechos humanos». Revista Redbioética, 1, (7): 25-34. https://ri.conicet.gov.ar/ handle/11336/12868

Bolaños, Angélica, Cerdas, Julandy y Quesada, Marilyn. 2017. «Violencia obstétrica en los servicios de salud de la Caja Costarricense del Seguro Social frente a los planteamientos de la “Guía de Atención Integral a las Mujeres, Niños y Niñas” en el Hospital Carlos Luis Valverde Vega en el período del 2014 al 2016». Seminario de graduación de licenciatura, Universidad de Costa Rica.

Borja, Carmen, García, Paloma e Hidalgo, Richard. 2011. El enfoque basado en derechos humanos: evaluación e indicadores. España: Red en Derechos y AECID. https://www.aecid.es/Centro-Documentacion/Documentos/Evaluaci\%C3\%B3n/ Eva luacion EBDH\%20+\%20NIPO+\%20logo.pdf

Camacaro, Marbella. 2008. «Dos sentencias masculinas, parirás con dolor... parirás acostada». En Mujeres en el mundo: Migración, género, trabajo, historia, arte y política, coordinado por Delgado, Yamile y González, María, 293- 310. Valencia, Venezuela: Editorial Markmedia Group, S. A.

Campos, Yerma. 2018. «Violencia obstétrica y su abordaje constitucional en Costa Rica». Revista Judicial, Poder Judicial de Costa Rica, 125: 41-61. ISSN 2215-2385.

Centro Nacional de Memoria Histórica. 2014. Desaparición forzada. Tomo III: Entre la incertidumbre y el dolor: impactos psicosociales de la desaparición forzada. Bogotá, Colombia: Imprenta Nacional. http://www.centrodememoriahistorica.gov.co /micrositios/ desaparicionForzada/libros-tomo3.html

Chaves, D. 2015. «Mujeres se unen en una marcha en contra de la violencia obstétrica». Radio Columbia versión digital. http://www.columbia.co.cr/index.php/ 
nacionales/11538-mujeres-se-unen-en-unamarcha-en-contra-de-la-violenciaobstetrica

Crenshaw, Kimberlé. 1989. «Demarginalizing the Intersection of Race and Sex: A Black Feminist Critique of Antidiscrimination Doctrine, Feminist Theory and Antiracist Politics». University of Chicago Legal Forum, 14: 139-167. https://chicagounbound.uchicago.edu/cgi/viewcontent.cgi $?$ article=1052\&context=uc If

De la Fuente, María. 2013. «Poder y feminismo: elementos para una teoría política». Tesis de doctorado en Ciencia Política y Derecho Público, Universidad de Barcelona.

De las Heras, Samara. 2009. «Una aproximación a las teorías feministas». Universitas. Revista de filosofía, derecho y política, (9): 45-82. http://universitas.idhbc.es/n09/0905.pdf

Dobles, Ignacio. y Rodríguez, D. 2019. Sobre el daño psicosocial: conceptualización y praxis. Manuscrito no publicado.

Ellsberg, Mary y Heise, Lori. 2007. Investigando la violencia contra las mujeres. Una guía práctica para la investigación y la acción. Managua, Nicaragua: Organización Mundial de la Salud y PATH.

Federici, Silvia. 2004. Calibán y la bruja. Mujeres, cuerpo y acumulación originaria. Madrid, España: Editorial Traficantes de sueños.

Fernández, Pablo. 2009. «Lo psicosocial». Revista desdisciplinada de psicología social, 2, (04): 41-48. http://elalmapublica.net/pdf/AP4.pdf

Fernández, Carmelo. 2013. «Sobre el concepto de patriarcado». Tesis de maestría, Universidad de Zaragoza. https://zaguan.unizar.es/record/10957 /files/TAZ-TFM2013-169.pdf

Foucault, Michel. 1998. Historia de la Sexualidad I. La voluntad del saber. Coyoacán, México: Siglo XXI Editores.

Fuentes, Eugenio. 2020. «Violencia obstétrica y su relación con determinantes sociales de la salud a partir de evidencia estadística en Costa Rica (2009-2011 y 2016-2018)». Tesis de maestría, Universidad de Costa Rica. 
García, Silvia. 2003. «Fertilizaciones cruzadas entre la Psicología Social de la Ciencia y los Estudios Feministas de la Ciencia». Revista Athenea Digital, 4: 1-42. http://ddd.uab.cat/pub/athdig/15788946n4/15788946n4a8.pdf

González, Fernando. 2004. «La crítica en la Psicología Social Latinoamericana y su impacto en los diferentes campos de la psicología». Revista Interamericana de Psicología, 38, (2): 351- 360. https://www.redalyc.org/articulo.oa?id=28438222

González, Mirta. 2010. «Feminismo, Feminismos. Avances hacia la equidad de género». En Teorías Psicosociales, compilado por González, Mirta, 115-153. San José, Costa Rica: Editorial Universidad de Costa Rica.

González, Mirta. 2012. Psicología Política. San José, Costa Rica: Editorial Universidad de Costa Rica.

González, Fernando. (2013). «La subjetividad en una perspectiva cultural-histórica: avanzando sobre un legado inconcluso». Revista CS, 11: 19-42. http://www.scielo.org.co/pdf/recs/n11/n11a02.pdf

Hendel, Liliana. 2017. Comunicación, infancia y adolescencia. Guía para periodistas. Perspectiva de género. Buenos Aires, Argentina: UNICEF. https://www.unicef.org/ argentina/sites/unicef.org.argentina/files/2018-04/COM-1_PerspectivaGenero_WE B.pdf

Hernández, Roberto, Fernández, Carlos y Baptista, Pilar. 2014. Metodología de la investigación. Sexta Edición. México D.F., México: Editorial McGraw-Hill. https://trabajosocial udocpno.files.wordpress.com/2017/07/metodologc3a3c2ada_de _la_investigacic3a3c2b3n_-sampieri-_6ta_edicion1.pdf

Hernández, Melissa. 2019. «Impacto psicosocial de la violencia obstétrica a partir de relatos de vida de mujeres». Tesis de licenciatura, Universidad de Costa Rica.

Herrera, Joaquín. 2008. «La complejidad de los derechos humanos: bases teóricas para una redefinición contextualizada». Revista Internacional de Direito e Cidadania, 1: 103135. http://www.ceapedi.com.ar/imagenes/biblioteca/libreria/338.pdf

Lagarde, Marcela. 2005. Los cautiverios de las mujeres: madresposas, monjas, putas, presas y locas. México D.F., México: Impreso por la Universidad Nacional Autónoma (UNAM).

Lerner, Gerda. 1989. La creación del Patriarcado. Barcelona, España: Editorial Crítica. 
Wimblu, Rev. Estud. de Psicología UCR, 16(2) 2021 (Julio-Diciembre): 93-118 /ISSN: 1659-2107

Machado, Alejandra. 2014. «¿Cómo parimos? De la violencia obstétrica al parto humanizado». Tesis de licenciatura, Universidad de la República. https://www.colibri.udelar.edu.uy/jspui/bitstream/123456789/5318/1/MACHADO.p df

Martín-Baró, Ignacio. 1990. Psicología social de la guerra. San Salvador, El Salvador: UCA Editores.

Martín-Baró, Ignacio. 2000. Acción e ideología. Psicología social desde Centroamérica. San Salvador, El Salvador: UCA Editores.

Morris, Krissia. 2015. «Da a luz en sanitario del Hospital de las Mujeres». Periódico Diario Extra, 06 de marzo. http://www.diarioextra.com/Noticia/detalle/254628/da-a-luz-ensanitario-delhospital-de-las-mujeres

Moscovici, Serge. 1985. Psicología Social I: Influencia y cambio de actitudes. Individuos y grupos. Buenos Aires, Argentina: Editorial Paidós.

Navarro, Óscar. (2012). Psicología social. Temas, teorías y aplicaciones. Medellín, Colombia: Imprenta Universidad De Antioquia.

Organización Mundial de la Salud. 1999. Dando prioridad a las mujeres: Recomendaciones éticas y de seguridad para la investigación sobre la violencia doméstica contra las mujeres. Ginebra, Suiza: OMS. https://www.who.int/violence_injury_prevention/ media/en/132.pdf

Organización Mundial de la Salud. 2014. Prevención y erradicación de la falta de respeto y el maltrato durante la atención al parto en centros de salud. Ginebra, Suiza: OMS. https://apps.who.int/iris/ bitstream/handle/10665/134590/WHO_RHR_14.23_spa.pdf;jsessionid=71C02E0B 2FE228009DCFE0509E530874? sequence=1

Oviedo, Steven. 2015. «Paciente denuncia violación a intimidad durante parto en Hospital de Las Mujeres». Periódico La Nación, 9 de marzo. http://www.nacion.com/nacional/ salud-publica/Mujerdenuncia-violacion-intimidad Carit_0_1474252667.html

Quevedo, Paula. (2012). «Violencia Obstétrica, una manifestación oculta de la desigualdad de género». Tesis de licenciatura, Universidad Nacional de Cuyo. https://bdigital.uncu.edu.ar/objetos_digitales/4785/tesisquevedo.pdf 
Ramírez, Alexánder. 2020. «Proyecto de ley contra la violencia obstétrica queda en firme. Congreso aprueba iniciativa en segundo debate». Crhoy, 13 de febrero. https://www.crhoy.com/nacionales/proyecto-de-ley-contra-violencia-obstetricaqueda-en-firme/

Restrepo, Andrés, Rodríguez, Daniela y Torres, Natalia. (2016). «"Me des-cuidaron el parto": La violencia obstétrica y el cuidado recibido por el personal de la salud a mujeres durante su proceso de parto». Tesis de licenciatura, Pontificia Universidad Javeriana. https://repository.javeriana.edu.co/handle/10554/20447

Robles, Keylor. 2018. «Visibilizando otras formas de violencia contra las mujeres: violencia obstétrica (2015)». Revista Estudiantil de Antropología Usuré, (I): 44-49.

Rodríguez, Ana y Vargas, Yishleny. 2017. «Expresiones del derecho a la salud en la atención pública a mujeres durante el proceso de embarazo en su vinculación con la violencia obstétrica». Tesis de licenciatura, Universidad de Costa Rica.

Ruíz, Paola. 2020. «Contra la violencia obstétrica: diputados aprueban 8 derechos para embarazadas». Observadorcr, 15 de febrero. https://observador.cr/noticia/contra-laviolencia-obstetrica-diputados-aprueban-8-derechos-para-embarazadas/

Salazar, Sharon y Torres, Yuliana. 2018. «Condiciones sociopolíticas existentes para la elaboración de una política pública que intervenga la violencia obstétrica en Costa Rica: una mirada desde el discurso, acciones y omisiones de diferentes actores sociales y políticos». Tesis de licenciatura, Universidad de Costa Rica.

Salaverry, Arabella. 2016. Breviario del deseo esquivo. $2^{\circ}$ edición. San José, Costa Rica: Editorial Costa Rica.

Salinas, Paulina y Cárdenas, Manuel. 2009. Métodos de investigación social. Quito, Ecuador: Editorial "Quipus", CIESPAL. https://biblio.flacsoandes.edu.ec/catalog/resGet. php?resId $=55376$

Segato, Rita. 2003. Las estructuras elementales de la violencia. Ensayos sobre género entre la antropología, el psicoanálisis y los derechos humanos. Buenos Aires, Argentina: Editorial Bernal, Universidad Nacional de Quilmes.

Simoes, Bruno. 2014. «Parecer técnico psicológico sobre Caso Reformatório Krenak». Inquérito Civil Público. 1.22.000.000929/2013-49. 


\section{ranu}

Wimblu, Rev. Estud. de Psicología UCR, 16(2) 2021 (Julio-Diciembre): 93-118 /ISSN: 1659-2107

Torres Ripa, Javier, adapt. y ed. 2013. Manual de estilo Chicago-Deusto. Bilbao, España: Publicaciones de la Universidad de Deusto.

Turégano, Isabel. 2009. «La violencia de género como vulneración de la dignidad humana: el papel del derecho en la lucha por la igual dignidad de la mujer». Revista Quid Iuris, 9: 103-122. http://historico.juridicas.unam.mx/publica/librev/rev/qdiuris/cont/9/cnt/ cnt5.pdf

Varela, Nuria. 2008. Feminismo para principiantes. Barcelona, España: Ediciones B, S.A. 